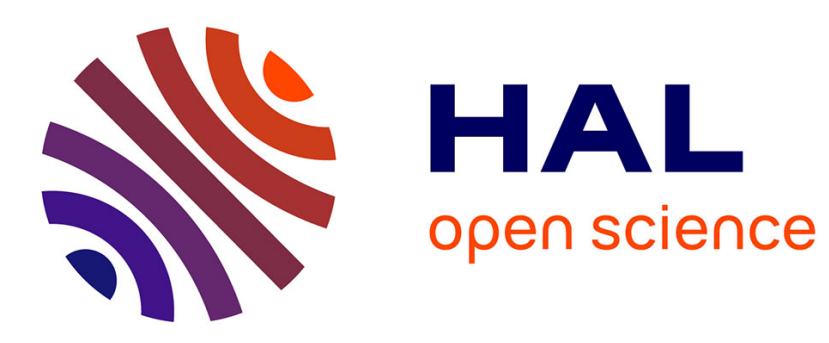

\title{
Fixed-time synchronization of competitive neural networks with multiple time-scale
}

Wu Yang, Yan-Wu Wang, Irinel-Constantin Morarescu, Xiao-Kang Liu, Yuehua Huang

\section{- To cite this version:}

Wu Yang, Yan-Wu Wang, Irinel-Constantin Morarescu, Xiao-Kang Liu, Yuehua Huang. Fixed-time synchronization of competitive neural networks with multiple time-scale. IEEE Transactions on Neural Networks and Learning Systems, 2021, pp.Early Access. 10.1109/TNNLS.2021.3052868 . hal03455236

\section{HAL Id: hal-03455236 \\ https://hal.science/hal-03455236}

Submitted on 29 Nov 2021

HAL is a multi-disciplinary open access archive for the deposit and dissemination of scientific research documents, whether they are published or not. The documents may come from teaching and research institutions in France or abroad, or from public or private research centers.
L'archive ouverte pluridisciplinaire HAL, est destinée au dépôt et à la diffusion de documents scientifiques de niveau recherche, publiés ou non, émanant des établissements d'enseignement et de recherche français ou étrangers, des laboratoires publics ou privés. 


\title{
Fixed-Time Synchronization of Competitive Neural Networks with Multiple Time-Scale
}

\author{
Wu Yang, Yan-Wu Wang, Senior Member, IEEE, Irinel-Constantin Morărescu, \\ Xiao-Kang Liu, and Yuehua Huang
}

\begin{abstract}
In this brief we investigate the fixed-time synchronization of competitive neural networks with multiple timescales. These neural networks play an important role in visual processing, pattern recognition, neural computing, etc. Our main contribution is the design of a novel synchronizing controller which does not depend on the ratio between the fast and slow time scales. This feature makes the controller easy to implement since it is designed through well-posed algebraic conditions (i.e. even when the ratio between the time scales goes to 0 the controller gain is well defined and does not go to infinity). Last but not least, the closed-loop dynamics is characterized by a high convergence speed with a settling time which is upper-bounded and the bound is independent of the initial conditions. A numerical simulation illustrates our results and emphasizes their effectiveness.
\end{abstract}

Index Terms-Competitive neural network, multiple time-scale feature, fixed-time synchronization, continuous control method.

\section{INTRODUCTION}

The concept of competitive neural networks with multiple time-scales (MTSCNNs) was introduced by Meyer-Bäse et al. [1] for modeling the dynamics of cortical cognitive maps. It is known that the cortex employs two types of memories: the long-term memory (LTM) and the short-term one. The longterm memory evolves on a slow time scale of unsupervised synaptic modifications while the short-term memory (STM) exhibits a much faster dynamics of the neural activity. It has been shown that such kind of neural networks play an important role in visual processing, pattern recognition, neural computing, and control [1]. Formally, MTSCNNs can be modeled as singularly perturbed systems whose processes evolve on multiple time scales [2].

One of the most fundamental problems in this context is the synchronization of competitive neural networks. Consequently, it received a lot of attention during the last decades. While some works consider dynamical models that evolves on one time scale [3]-[7] some others go further and propose models evolving on two time scales. Different scenarios addressing

This work is supported by the National Natural Science Foundation of China under Grants 61903147 and 61773172, and the Fundamental Research Funds for the Central Universities (2018KFYYXJJ119). (Corresponding author: Xiao-Kang Liu.)

Wu Yang, Yan-Wu Wang, and Xiao-Kang Liu are with the Key Laboratory of Image Processing and Intelligent Control, School of Artificial Intelligence and Automation, Huazhong University of Science and Technology, Wuhan, China, 430074. Wu Yang is also with the School of Electrical Engineering, Guangxi University, Nanning 530004, China. (e-mail: wangyw@ hust.edu.cn).

Irinel-Constantin Morărescu is with Université de Lorraine, CNRS, CRAN, Nancy, 54000, France.

Yuehua Huang is with College of Electrical Engineering and New Energy, China Three Gorges University, Yichang, 443002, China. synchronization of MTSCNNs in presence of stochastic perturbations, hybrid perturbations, fixed or time-varying delays [8][14] can be found in the literature. The main inconvenient of these results is that the derived synchronization criteria or the proposed controller' parameters depend on the ratio between the fast and slow time-scales. Consequently, when this ratio approaches 0 the problem becomes ill-conditioned. Moreover, all aforementioned works focus solely on asymptotic synchronization, i.e., synchronization can be ensured in infinite time, which may be insufficient in applications. We also notice the existence of few results on the finite-time synchronization of MTSCNNs [15]-[17]. It is noteworthy that the settling-time provided by finite-time synchronization heavily depends on the initial conditions of the network (see Table I in Section IV). Consequently, two obvious shortcomings of those works are: 1) the settling-time may be very large for some initial conditions; 2) the settling time cannot be estimated in advance since the initial conditions are generally unknown.

To overcome the inconvenient of the finite-time synchronization, the fixed-time synchronization was introduced by extending the concept of fixed-time stability (see [18], [19] for details). In particular, the settling-time of the fixed-time synchronization does not depend on the initial conditions of the network [20], [21].

In this context, we propose to go further and design a controller that ensures the fixed-time synchronization of MTSCNNs. The main difficulties of the proposed methodology are threefold: 1) the results on fixed-time synchronization of regular neural networks proposed in [20], [21] cannot be directly applied here since they lead to ill-conditioning (see [2] for details); 2) the synchronization problem at hand is challenging since the slow and fast coupled dynamics are both nonlinear; 3 ) the controller achieving fixed time synchronization has to be implementable and well defined i.e. independent on the ration between the slow and fast time scales. Note that the above difficulties are not present when dealing with one time scale dynamics. This renders our study interesting but very challenging.

The main contributions of this brief are the following: we design an appropriate control mechanism that achieves fixedtime synchronization of MTSCNNs; we render the proposed controller implementable and well-conditioned by designing it independent of the ratio between the slow and fast time-scales; we characterize the relation between the controller' parameters and the settling-time.

Notations: We use the following standard notations. $\mathbb{R}$ and $\mathbb{R}^{n}$ represent the set of real numbers and the vector space of 
real $n$ dimensional vectors, respectively; $\operatorname{sign}(\cdot)$ is the function equals 1 if its argument is positive, -1 if its argument is negative and 0 if its argument is $0 .|a|$ denotes the absolute value of the constant $a,\|x\|_{\sigma}$ denotes the $\sigma$-norm of the vector $x \in \mathbb{R}^{n}$, where $\sigma \in\{1,2\} . A^{T}$ denotes the transpose of the matrix $A$. For a set of column vectors $x_{1}, x_{2}, \cdots, x_{n}$, $\operatorname{col}\left(x_{1}, x_{2}, \cdots, x_{n}\right)=\left[\begin{array}{llll}x_{1}^{T} & x_{2}^{T} & \cdots, & x_{n}^{T}\end{array}\right]^{T}$. We say that function $f(t, \varepsilon):[0, \infty) \times[0, \infty) \mapsto R^{m \times n}$ is $O(\varepsilon)$, if there exist positive constants $k$ and $\varepsilon^{*}$ strictly positive such that $\|f(t, \varepsilon)\| \leq k \varepsilon$ for all $t \in[0, \infty)$ and $\varepsilon \in\left[0, \varepsilon^{*}\right]$.

\section{Model Description AND Preliminaries}

In this section, we present the model of a class of MTSCNNs and give some useful lemmas and definition.

Consider the following MTSCNNs:

$$
\mathrm{STM}: \varepsilon \dot{x}_{i}(t)=-a_{i} x_{i}(t)+\sum_{j=1}^{N} b_{i j} f_{j}\left(x_{j}(t)\right)+d_{i} S_{i}(t),
$$

$$
\text { LTM : } \dot{S}_{i}(t)=-S_{i}(t)+f_{i}\left(x_{i}(t)\right) .
$$

where $i=1,2, \cdots, N$, with $N$ being the number of neurons, $x_{i}(t), a_{i}$, and $f_{i}\left(x_{i}(t)\right)$ are the current activity level, the time constant, and the activation function of the $i$-th neuron, respectively. The contribution of the external stimulus is denoted by $d_{i}$ and $S_{i}(t)=\sum_{j=1}^{Q} m_{i j}(t) I_{j}, I_{j}(j=1,2, \cdots, Q)$ is the external stimulus, where $Q$ is the number of the external stimulus, $m_{i j}$ is the synaptic efficiency, and $\varepsilon$ is the time scale parameter associated with the STM state (i.e., $\varepsilon$ is the ratio between the fast and slow time-scales). $b_{i j}$ denotes the connection weight strength between the $i$-th neuron and the $j$-th neuron. The initial condition of network (1)-(2) is given as $x_{i}\left(t_{0}\right)=x_{i 0}, S_{i}\left(t_{0}\right)=S_{i 0}$. The following assumption is given for the activation function $f_{i}(\cdot)$.

Assumption 1: [3], [5], [8] The activation function $f_{i}(\cdot), i=$ $1,2, \cdots, N$, is Lipschitz continuous with the Lipschitz constant $L_{i}>0$, that is,

$$
\left|f_{i}(u)-f_{i}(v)\right| \leq L_{i}|u-v|,
$$

for all $u, v \in \mathbb{R}$.

The activation function $f_{i}(\cdot)$ in Assumption 1 is more general than the usual ones i.e. the sigmoid activation functions and the piecewise linear function $f_{i}(s)=0.5(|s+1|-|s-1|)$.

Based on the concept of master-slave synchronization [22], network (1)-(2) is seen as the master network and the corresponding controlled slave one is described as follows:

$$
\begin{aligned}
\varepsilon \dot{y}_{i}(t) & =-a_{i} y_{i}(t)+\sum_{j=1}^{N} b_{i j} f_{j}\left(y_{j}(t)\right)+d_{i} R_{i}(t)+u_{i}(t) \\
\dot{R}_{i}(t) & =-R_{i}(t)+f_{i}\left(y_{i}(t)\right)+v_{i}(t)
\end{aligned}
$$

with the initial condition $y_{i}\left(t_{0}\right)=y_{i 0}$ and $R_{i}\left(t_{0}\right)=R_{i 0}$, where $y_{i}(t)$ and $R_{i}(t)$ are the state variables of the slave network (4)-(5), $u_{i}(t)$ and $v_{i}(t)$ are the controllers to be designed for the STM (4) and the LTM (5), respectively. The other parameters are the same as in network (1)-(2). Let us define the synchronization error $z_{i}(t)=y_{i}(t)-x_{i}(t)$ and $w_{i}(t)=R_{i}(t)-S_{i}(t)$. With this notation at hand we obtain the following synchronization error dynamics:

$$
\begin{aligned}
\varepsilon \dot{z}_{i}(t) & =-a_{i} z_{i}(t)+\sum_{j=1}^{N} b_{i j} \tilde{f}_{j}\left(z_{j}(t)\right)+d_{i} w_{i}(t)+u_{i}(t), \\
\dot{w}_{i}(t) & =-w_{i}(t)+\tilde{f}_{i}\left(z_{i}(t)\right)+v_{i}(t) .
\end{aligned}
$$

where $\tilde{f}\left(z_{i}(t)\right)=f\left(y_{i}(t)\right)-f\left(x_{i}(t)\right)$. The initial condition of (6) and (7) is $z_{i}\left(t_{0}\right)=x_{i 0}-y_{i 0}, w_{i}\left(t_{0}\right)=S_{i 0}-R_{i 0}$.

The main objective of this brief is to provide new results on the global fixed-time synchronization between network (4)(5) and network (1)-(2). Before moving on, we provide few lemmas and definitions.

Definition 1: [18] Consider the nonlinear systems

$$
\dot{x}=g(t, x), x(0)=x_{0},
$$

where $x \in \mathbb{R}^{n}$ and $g: \mathbb{R}^{+} \times \mathbb{R}^{n} \mapsto \mathbb{R}^{n}$ is a nonlinear function. The origin of (8) is said to be globally finite-time stable if it is globally asymptotically stable and any solution $x\left(t, x_{0}\right)$ of (8) reaches the equilibrium at some finite time that depends on the initial condition, i.e., $x\left(t, x_{0}\right)=0, \forall t \geq T\left(x_{0}\right)$. Moreover, the origin of (8) is said to be globally fixed-time stable if it is globally finite-time stable and the settling-time function $T\left(x_{0}\right)$ is bounded, i.e., there exists $T_{\max }$ such that $T\left(x_{0}\right) \leq T_{\max }$ for any $x_{0} \in \mathbb{R}^{n}$.

Lemma 1: [18], [20] For the nonnegative scalars $\theta_{i}, i=$ $1,2, \cdots, s, p \in(0,1]$, and $q>1$, the following inequalities hold,

$$
\sum_{i=1}^{s} \theta_{i}^{p} \geq\left(\sum_{i=1}^{s} \theta_{i}\right)^{p}, \sum_{i=1}^{s} \theta_{i}^{q} \geq s^{1-q}\left(\sum_{i=1}^{s} \theta_{i}\right)^{q} .
$$

Lemma 2: [18], [20] Consider the nonlinear system $\dot{\chi}(t)=$ $g(\chi(t)), \chi\left(t_{0}\right)=\chi_{0}$, where $\chi(t) \in \mathbb{R}^{n}$ denotes the state variable, and $\chi_{0}$ is the initial state. If there exists a continuous radially unbounded function $V: \mathbb{R}^{n} \mapsto \mathbb{R}^{+} \cup\{0\}$ such that

1) $V(\chi)=0 \Leftrightarrow \chi=0$;

2) for some $\alpha, \beta>0,0<p<1$, and $q>1$, any solution $z(t)$ satisfies the inequality

$$
\dot{V}(\chi(t)) \leq-\alpha V^{p}(\chi(t))-\beta V^{q}(\chi(t)),
$$

then the origin of the system under consideration is globally fixed-time stable, that is,

$$
V(\chi(t)) \equiv 0, t \geq T_{\max }=\frac{1}{\alpha(1-p)}+\frac{1}{\beta(q-1)}, \forall \chi_{0} \in \mathbb{R}^{n} .
$$

Definition 2: Given $\varepsilon^{*}>0$, network (4)-(5) is said to achieve the global fixed-time synchronization with network (1)-(2) for $\varepsilon \in\left(0, \varepsilon^{*}\right)$ if there exists a settling-time $T_{\max }\left(\varepsilon^{*}\right)>0$, which is independent on the initial value, such that for any initial value $z_{i}\left(t_{0}\right)$ and $w_{i}\left(t_{0}\right)$,

$$
\begin{aligned}
& \lim _{t \rightarrow T_{\max }}\left\|z_{i}(t)\right\|=0, \lim _{t \rightarrow T_{\max }}\left\|w_{i}(t)\right\|=0, \forall i \in \mathcal{N}, \\
& z_{i}(t) \equiv 0, w_{i}(t) \equiv 0, \forall t \geq T_{\max }\left(\varepsilon^{*}\right) .
\end{aligned}
$$

Remark 1: Definition 2 requires that the fixed-time synchronization of MTSCNNs can be maintained for any $\varepsilon \in\left(0, \varepsilon^{*}\right)$ 
with the given positive constant $\varepsilon^{*}$. Although it may seem restrictive, it is reasonable to have the settling-time $T_{\max }\left(\varepsilon^{*}\right)$ depending on $\varepsilon^{*}$. Our results state that, once $\varepsilon^{*}$ is given, one can design an implementable controller that synchronizes the MTSCNNs in less than $T_{\max }$ time.

\section{FiXed-Time Synchronization AnALysis}

Note that the fixed-time synchronization between networks (4)-(5) and (1)-(2) can be converted into the fixed-time stabilization of the synchronization error dynamics (6)-(7). In this section, we firstly present a generalized fixed-time stability result of singularly perturbed systems. This allows us to treat the dynamics (6)-(7) as a special case. Next, we design the fixed-time synchronization controller and analyze the synchronization of the networks under consideration.

Let us consider the following generic nonlinear singularly perturbed system:

$$
\left\{\begin{array}{l}
\dot{x}(t)=f(x(t), z(t)), \quad x\left(t_{0}\right)=x_{0}, \\
\varepsilon \dot{z}(t)=g(x(t), z(t)), \quad z\left(t_{0}\right)=z_{0},
\end{array}\right.
$$

where $x(t) \in \mathbb{R}^{n_{x}}$ is the slow state vector, $z(t) \in \mathbb{R}^{n_{z}}$ is the fast state vector, $\varepsilon$ is a small parameter that characterizes the time-scale separation between the fast and slow dynamics. $f: \mathbb{R}^{n_{x}} \times \mathbb{R}^{n_{z}} \rightarrow \mathbb{R}^{n_{x}}$ and $g: \mathbb{R}^{n_{x}} \times \mathbb{R}^{n_{z}} \rightarrow \mathbb{R}^{n_{z}}$ are nonlinear functions with $f(0,0)=0, g(0,0)=0$. The origin $(x=0, z=0)$ is assumed to be an isolated equilibrium point for system (12). Given $\varepsilon^{*}>0$, system (12) is said globally fixed-time stable for any $\varepsilon \in\left(0, \varepsilon^{*}\right)$ if there exists a settling time $T_{\max }\left(\varepsilon^{*}\right)>0$, which is independent of the initial value, such that $\lim _{t \rightarrow T_{\max }\left(\varepsilon^{*}\right)}\|x(t)\|_{\sigma}+\|z(t)\|_{\sigma}=0, \sigma \in\{1,2\}$ and $x(t) \equiv 0, z(t) \equiv 0, \forall t \geq T_{\max }\left(\varepsilon^{*}\right)$ hold for any initial values $x_{0}$ and $z_{0}$.

Now, based on the above preliminaries, the main result on globally fixed-time stability of system (12) is given.

Lemma 3: Given $\varepsilon^{*}>0$, system (12) is globally fixed-time stable if there exists a composite function $V(t)=U_{1}(x(t))+$ $\varepsilon U_{2}(z(t))$ such that for any $\sigma \in\{1,2\}$

$$
\begin{aligned}
k_{1}\|x\|_{\sigma}^{2} \leq & U_{1}(x(t)) \leq k_{2}\|x\|_{\sigma}^{2}, \\
k_{3}\|z\|_{\sigma}^{2} \leq & U_{2}(z(t)) \leq k_{4}\|z\|_{\sigma}^{2}, \\
\left.\dot{V}(t)\right|_{(12)} \leq & -\alpha\left(\|x\|_{\sigma}^{p}+\|z\|_{\sigma}^{p}\right) \\
& -\beta\left(\|x\|_{\sigma}^{1+q}+\|z\|_{\sigma}^{1+q}\right),
\end{aligned}
$$

where $k_{i}>0, i=1,2,3,4, \alpha>0, \beta>0, p \in(0,1), q>1$, and the settling-time is upper-bounded by

$T_{\max }\left(\varepsilon^{*}\right)=\underbrace{\frac{2\left(k_{2}\right)^{\frac{p}{2}}}{\alpha(2-p)}+\frac{\left(2 k_{2}\right)^{\frac{1+q}{2}}}{\beta(q-1)}}_{T_{\max }^{1}}+\underbrace{\frac{2\left(\varepsilon^{*} k_{4}\right)^{\frac{p}{2}}}{\alpha(2-p)}+\frac{\left(2 \varepsilon^{*} k_{4}\right)^{\frac{1+q}{2}}}{\beta(q-1)}}_{T_{\max }^{*}}$

Proof: It follows from Lemma 1 and conditions (13)-(14) that

$$
\begin{aligned}
& 2^{\frac{1-q}{2}}[V(t)]^{\frac{1+q}{2}} \leq 2^{\frac{1-q}{2}}\left[k_{2}\|x\|_{\sigma}^{2}+\varepsilon k_{4}\|z\|_{\sigma}^{2}\right]^{\frac{1+q}{2}} \\
& \leq\left[\left(k_{2}\right)^{\frac{1+q}{2}}+\left(\varepsilon k_{4}\right)^{\frac{1+q}{2}}\right]\left(\|x\|_{\sigma}^{1+q}+\|z\|_{\sigma}^{1+q}\right),
\end{aligned}
$$

and

$$
\begin{aligned}
{[V(t)]^{\frac{p}{2}} } & \leq\left[k_{2}\|x\|_{\sigma}^{2}+\varepsilon k_{4}\|z\|_{\sigma}^{2}\right]^{\frac{p}{2}} \\
& \leq\left[\left(k_{2}\right)^{\frac{p}{2}}+\left(\varepsilon k_{4}\right)^{\frac{p}{2}}\right]\left(\|x\|_{\sigma}^{p}+\|z\|_{\sigma}^{p}\right) .
\end{aligned}
$$

Then, condition (15) implies

$$
\begin{aligned}
\dot{V}(t) \leq & -\frac{\alpha}{\left(k_{2}\right)^{\frac{p}{2}}+\left(\varepsilon k_{4}\right)^{\frac{p}{2}}}[V(t)]^{\frac{p}{2}} \\
& -\frac{2^{\frac{1-q}{2}} \beta}{\left(k_{2}\right)^{\frac{1+q}{2}}+\left(\varepsilon k_{4}\right)^{\frac{1+q}{2}}}[V(t)]^{\frac{1+q}{2}} .
\end{aligned}
$$

Finally, according to Lemma 2, it can be obtained that $V(t) \equiv$ $0, \forall t \geq T_{\max }\left(\varepsilon^{*}\right)$, where $T_{\max }\left(\varepsilon^{*}\right)$ is given by (16).

Remark 2: Reference [18] establishes the fixed-time stability condition for a general nonlinear systems. In this context, a discussion highlighting the usefulness of Lemma 3 is required.

1) Multiple time-scales feature of singularly perturbed systems hampers the application of the results in [18]. One of the main contribution of Lemma 3 is that it construct an $\varepsilon$-dependent composite Lyapunov function, in which Lyapunov functions $U_{1}(x(t))$ and $U_{2}(z(t))$ are specifically designed for analyzing the slow and the fast dynamics, respectively;

2) The presence of the singular perturbation parameter introduces supplementary difficulties in the estimation of the settling-time. It is noteworthy that the results in [18] are not tailored for this situation. Moreover, Lemma 3 reveals how the singular perturbation parameter $\varepsilon$ affects the settling-time. To be more precise, the settlingtime is the sum of two terms $T_{\max }^{1}$ and $T_{\max }^{\varepsilon}$, where $T_{\max }^{\varepsilon}$ depends on the parameter $\varepsilon$. It is noteworthy that Lemma 3 requires the parameter $\varepsilon$ to be sufficiently small although it is unknown. In other words, it also can be concluded that system (12) is globally fixed-time stable as far as $\varepsilon<\varepsilon^{*}$. In this case, the settling-time is approximated by $T_{\max }^{1}$ with an $O\left(\varepsilon^{\frac{p}{2}}\right)$ error. Moreover, when $\varepsilon \rightarrow 0^{+}, T_{\max }^{1}$, determined by the parameters of the slow dynamics, plays a dominant role in the settlingtime.

To achieve the globally fixed-time stability of the synchronization error network (6)-(7), the following controllers $u_{i}(t)$ and $v_{i}(t)$ are designed:

$$
\begin{aligned}
& u_{i}(t)=-\rho_{i} z_{i}(t)-\gamma_{i}\left(\left\lceil z_{i}(t)\right\rfloor^{p}+\left\lceil z_{i}(t)\right\rfloor^{q}\right), \\
& v_{i}(t)=-\eta_{i} w_{i}(t)-\gamma_{i}\left(\left\lceil w_{i}(t)\right\rfloor^{p}+\left\lceil w_{i}(t)\right\rfloor^{q}\right),
\end{aligned}
$$

where $\lceil s\rfloor^{\kappa}=|s|^{\kappa} \operatorname{sign}(s)$. Note that $-\rho_{i} z_{i}(t)$ and $-\eta_{i} w_{i}(t)$ are used to achieve the asymptotic stability of dynamics (6)(7). The terms $-\gamma_{i}\left\lceil z_{i}(t)\right\rfloor^{p}$ and $-\gamma_{i}\left\lceil w_{i}(t)\right\rfloor^{p}$ are introduced to speed-up the convergence and achieve the finite-time stability of the error dynamics (6)-(7). Finally, the terms $-\gamma_{i}\left\lceil z_{i}(t)\right\rfloor^{q}$ and $-\gamma_{i}\left\lceil w_{i}(t)\right\rfloor^{q}$ play a decisive role in achieving the fixedtime stability.

Remark 3: Note that the synchronization mechanisms designed in [8]-[14] can only achieve the asymptotic synchronization while the controllers in [15]-[17] achieve the finite-time synchronization of MTSCNNs. The synchronizing controller (20)-(21) is continuous and can ensure the fixedtime synchronization of MTSCNNs. 
With all these preliminaries at hand, we are ready to present the main results on the global fixed-time synchronization of the networks (4)-(5) and (1)-(2).

Theorem 1: Under Assumption 1 suppose that for a given $\varepsilon^{*}>0$ the design parameters of the controller (20) and (21) are selected as follows:

$$
\begin{aligned}
& \rho_{i} \geq 0.5\left[\sum_{j=1}^{N}\left(\left|b_{i j}\right| L_{j}+\left|b_{j i}\right| L_{i}\right)+L_{i}+\left|d_{i}\right|\right]-a_{i}, \\
& \eta_{i} \geq 0.5\left(L_{i}+\left|d_{i}\right|\right)-1, \gamma_{i}>0, p \in(0,1), q>1 .
\end{aligned}
$$

Then, the global fixed-time synchronization between network (4)-(5) and network (1)-(2) can be guaranteed, and the settling time $T_{1}\left(\varepsilon^{*}\right)$ is computed by

$$
\frac{1+\left(\varepsilon^{*}\right)^{\frac{p+1}{2}}}{\check{\gamma}(1-p)}+\frac{(2 N)^{\frac{q-1}{2}}\left[1+\left(\varepsilon^{*}\right)^{\frac{q+1}{2}}\right]}{\check{\gamma}(q-1)},
$$

where $\check{\gamma}=\min \left\{\gamma_{i}, i=1,2, \cdots, N\right\}$.

Proof: Let us choose the following composite Lyapunov candidate $V_{1}(t)=\sum_{i=1}^{N}\left[\varepsilon z_{i}^{2}(t)+w_{i}^{2}(t)\right]$. The time derivative of $V_{1}(t)$ along the trajectories of network (6)-(7) is given by

$$
\begin{aligned}
& \dot{V}_{1}(t)=2 \sum_{i=1}^{N}\left[z_{i}(t) \varepsilon \dot{z}_{i}(t)+w_{i}(t) \dot{w}_{i}(t)\right] \\
& =-2 \sum_{i=1}^{N}\left(\rho_{i}+a_{i}\right) z_{i}^{2}(t)+2 \sum_{i=1}^{N} \sum_{j=1}^{N} b_{i j} z_{i}(t) \tilde{f}_{j}\left(z_{j}(t)\right) \\
& +2 \sum_{i=1}^{N} z_{i}(t) d_{i} w_{i}(t)-2 \sum_{i=1}^{N} \gamma_{i}\left(\left|z_{i}(t)\right|^{p+1}+\left|z_{i}(t)\right|^{q+1}\right) \\
& -2 \sum_{i=1}^{N}\left(\eta_{i}+1\right) w_{i}^{2}(t)+2 \sum_{i=1}^{N} w_{i}(t) \tilde{f}_{i}\left(z_{i}(t)\right) \\
& -2 \sum_{i=1}^{N} \gamma_{i}\left(\left|w_{i}(t)\right|^{p+1}+\left|w_{i}(t)\right|^{q+1}\right) .
\end{aligned}
$$

It follows from Assumption 1 that

$$
\begin{aligned}
2 \sum_{i=1}^{N} & \sum_{j=1}^{N} b_{i j} \tilde{f}_{j}\left(z_{j}(t)\right) \leq 2 \sum_{i=1}^{N} \sum_{j=1}^{N}\left|b_{i j}\right| L_{j}\left|z_{i}(t)\right|\left|z_{j}(t)\right| \\
& =\sum_{i=1}^{N} \sum_{j=1}^{N}\left(\left|b_{i j}\right| L_{j}+\left|b_{j i}\right| L_{i}\right) z_{i}^{2}(t)
\end{aligned}
$$

$$
\begin{aligned}
2 \sum_{i=1}^{N} w_{i}(t) \tilde{f}_{i}\left(z_{i}(t)\right) & \leq \sum_{i=1}^{N} L_{i}\left(w_{i}^{2}(t)+z_{i}^{2}(t)\right), \\
2 \sum_{i=1}^{N} z_{i}(t) d_{i} w_{i}(t) & \leq \sum_{i=1}^{N}\left|d_{i}\right|\left(w_{i}^{2}(t)+z_{i}^{2}(t)\right) .
\end{aligned}
$$

From (25)-(28), we derive

$$
\begin{aligned}
\dot{V}_{1}(t) \leq & -2 \check{\gamma} \sum_{i=1}^{N}\left(\left|z_{i}(t)\right|^{p+1}+\left|w_{i}(t)\right|^{p+1}\right) \\
& -2 \check{\gamma} \sum_{i=1}^{N}\left(\left|z_{i}(t)\right|^{q+1}+\left|w_{i}(t)\right|^{q+1}\right),
\end{aligned}
$$

by using conditions (22) and (23) and the notations $\check{\gamma}=$ $\min \left\{\gamma_{i}, i=1,2, \cdots, N\right\}$.

By using Lemma 1, it follows that

$$
\begin{array}{r}
{\left[V_{1}(t)\right]^{\frac{p+1}{2}} \leq\left(1+\varepsilon^{\frac{p+1}{2}}\right) \sum_{i=1}^{N}\left[\left|z_{i}(t)\right|+\left|w_{i}(t)\right|\right],} \\
{\left[V_{1}(t)\right]^{\frac{q+1}{2}} \leq \frac{1+\varepsilon^{\frac{q+1}{2}}}{(2 N)^{\frac{1-q}{2}}} \sum_{i=1}^{N}\left[\left|z_{i}(t)\right|^{q+1}+\left|w_{i}(t)\right|^{q+1}\right] .}
\end{array}
$$

Substituting (30) and (31) into (36), we then have

$$
\dot{V}_{1}(t) \leq-\frac{2 \check{\gamma}}{1+\varepsilon^{\frac{p+1}{2}}}\left[V_{1}(t)\right]^{\frac{p+1}{2}}-\frac{2 \check{\gamma}(2 N)^{\frac{1-q}{2}}}{1+\varepsilon^{\frac{q+1}{2}}}\left[V_{1}(t)\right]^{\frac{q+1}{2}} .
$$

Based on Lemma 2, it can be concluded that $V_{1}(t) \equiv 0$ as $t \rightarrow T_{1}(\varepsilon)$, which is given by

$T_{1}(\varepsilon)=\frac{1}{\check{\gamma}(1-p)}+\frac{(2 N)^{\frac{q-1}{2}}}{\check{\gamma}(q-1)}+\frac{\varepsilon^{\frac{p+1}{2}}}{\check{\gamma}(1-p)}+\frac{(2 N)^{\frac{q-1}{2}} \varepsilon^{\frac{1+q}{2}}}{\check{\gamma}(q-1)}$.

Note that the settling time $T_{1}(\varepsilon)$ is monotonically increasing with respect to $\varepsilon$, which further means that the networks (4)-(5) and (1)-(2) are globally fixed-time synchronized for $\varepsilon \in\left(0, \varepsilon^{*}\right)$ via the controller (20)-(21) and the most conservative of the settling time is given by (24). The proof is thus completed.

If we take the composite Lyapunov candidate $V_{2}(t)=$ $\varepsilon\|z(t)\|_{1}+\|w(t)\|_{1}=\sum_{i=1}^{N}\left[\varepsilon\left|z_{i}(t)\right|+\left|w_{i}(t)\right|\right]$, we have the following result.

Theorem 2: Under Assumption 1 suppose that for a given $\varepsilon^{*}>0$ the design parameters of the controller (20) and (21) are selected as follows:

$$
\begin{aligned}
& \rho_{i} \geq \sum_{j=1}^{N}\left|b_{j i}\right| L_{i}+L_{i}-a_{i}, \\
& \eta_{i} \geq\left|d_{i}\right|-1, \gamma_{i}>0, p \in(0,1), q>1 .
\end{aligned}
$$

Then, the global fixed-time synchronization between network (4)-(5) and network (1)-(2) is guaranteed, and the settling time is upper-bounded by

$$
T_{2}\left(\varepsilon^{*}\right)=\frac{1+\left(\varepsilon^{*}\right)^{p}}{\check{\gamma}(1-p)}+\frac{(2 N)^{q-1}\left(1+\left(\varepsilon^{*}\right)^{q}\right)}{\check{\gamma}(q-1)} .
$$

Proof: The time derivative of $V_{2}(t)$ along the trajectories of network (6)-(7) is given by

$$
\begin{aligned}
& \dot{V}_{2}(t)=\sum_{i=1}^{N}\left[\operatorname{sign}\left(z_{i}(t)\right) \varepsilon \dot{z}_{i}(t)+\operatorname{sign}\left(w_{i}(t)\right) \dot{w}_{i}(t)\right] \\
& \leq-\sum_{i=1}^{N}\left(\rho_{i}+a_{i}\right)\left|z_{i}(t)\right|+\sum_{i=1}^{N} \sum_{j=1}^{N}\left|b_{i j}\right| L_{j}\left|z_{j}(t)\right| \\
& +\sum_{i=1}^{N}\left(\left|d_{i}\right|-1-\eta_{i}\right)\left|w_{i}(t)\right|+\sum_{i=1}^{N} L_{i}\left|z_{i}(t)\right|-\sum_{i=1}^{N} \gamma_{i}\left|z_{i}(t)\right|^{p} \\
& -\sum_{i=1}^{N} \gamma_{i}\left|z_{i}(t)\right|^{q}-\sum_{i=1}^{N} \gamma_{i}\left|w_{i}(t)\right|^{p}-\sum_{i=1}^{N} \gamma_{i}\left|w_{i}(t)\right|^{q} \\
& \leq-\check{\gamma} \sum_{i=1}^{N}\left(\left|z_{i}(t)\right|^{p}+\left|w_{i}(t)\right|^{p}\right)-\check{\gamma} \sum_{i=1}^{N}\left(\left|z_{i}(t)\right|^{q}+\left|w_{i}(t)\right|^{q}\right) .
\end{aligned}
$$


Note that

$$
\begin{aligned}
{\left[V_{2}(t)\right]^{p} } & =\left(\sum_{i=1}^{N} \varepsilon\left|z_{i}(t)\right|+\left|w_{i}(t)\right|\right)^{p} \\
& \leq\left(1+\varepsilon^{p}\right) \sum_{i=1}^{N}\left(\left|z_{i}(t)\right|^{p}+\left|w_{i}(t)\right|^{p}\right),
\end{aligned}
$$

and

$$
\begin{aligned}
(2 N)^{1-q}\left[V_{2}(t)\right]^{q} & =(2 N)^{1-q}\left(\sum_{i=1}^{N} \varepsilon\left|z_{i}(t)\right|+\left|w_{i}(t)\right|\right)^{q} \\
& \leq\left(1+\varepsilon^{q}\right) \sum_{i=1}^{N}\left(\left|z_{i}(t)\right|^{q}+\left|w_{i}(t)\right|^{q}\right),
\end{aligned}
$$

Substituting (30) and (31) into (36), we then have

$$
\dot{V}_{2}(t) \leq-\frac{\check{\gamma}}{1+\varepsilon^{p}}\left[V_{2}(t)\right]^{p}-\frac{\check{\gamma}(2 N)^{1-q}}{1+\varepsilon^{q}}\left[V_{2}(t)\right]^{q} .
$$

Based on Lemma 2, it can be concluded that $V_{2}(t) \equiv 0$ as $t \rightarrow T_{2}(\varepsilon)$, which is given by $T_{2}(\varepsilon)=\frac{1+\varepsilon^{p}}{\check{\gamma}(1-p)}+\frac{(2 N)^{q-1}\left(1+\varepsilon^{q}\right)}{\check{\gamma}(q-1)}$. Then, it is easy to draw the conclusion that the networks (4)(5) and (1)-(2) is globally fixed-time synchronized. The proof is thus completed.

Remark 4: The upper-bounds on the settling-time provided in Theorem 1 and 2 do not depend on the initial conditions of the networks. These values depend on the design parameters. Although the settling-time depends on the parameter $\varepsilon$, Theorems 1 and 2 show that they are upper-bounded by values that depend only on $\varepsilon^{*}$. Moreover, we have $T_{1}\left(\varepsilon^{*}\right) \leq T_{2}\left(\varepsilon^{*}\right)$ for the same $\gamma_{i}, p$, and $q$.

\section{Simulation}

In this section, a simulation example is given to show the efficiency of the obtained results.

Example 1: Consider the following 4-dimensional MTSCNNs of the form (1)-(2) with the model parameters

$$
\begin{aligned}
& a_{1}=1, b_{11}=0.5, b_{12}=-1, d_{1}=1.2, \\
& a_{2}=0.5, b_{21}=0.6, b_{22}=0.5, d_{2}=1.2, \varepsilon=0.05, \\
& f_{1}(s)=\left\{\begin{array}{l}
s+\sin (s+1)-1, s<-1, \\
2 s,-1 \leq s \leq 1, \\
s+\sin (s-1)+1, s>1,
\end{array}\right. \\
& f_{2}(s)=\left\{\begin{array}{l}
2 s+\cos (s+1)+2, s<-1, \\
s,-1 \leq s \leq 1, \\
2 s+\cos (s-1)-2, s>1 .
\end{array}\right.
\end{aligned}
$$

Then Assumption 1 holds for $L_{1}=2$ and $L_{2}=3$. Notice that the activation functions $f_{i}(\cdot), i=1,2$ are unbounded. The time evolution of the states of MTSCNNs described in Example 1 is given in Fig. 1. This shows clearly that the MTSCNN has a periodic solution.

As explained in the introduction and Remark 3, the methods in [8]-[17] cannot be employed to achieve the fixed-time synchronization of MTSCNNs of Example 1.

To illustrate the effectiveness of our results we define the synchronization error index $E(t)=\sum_{i=1}^{2}\left[\left|z_{i}(t)\right|+\left|w_{i}(t)\right|\right]$ and set the initial states as follows:

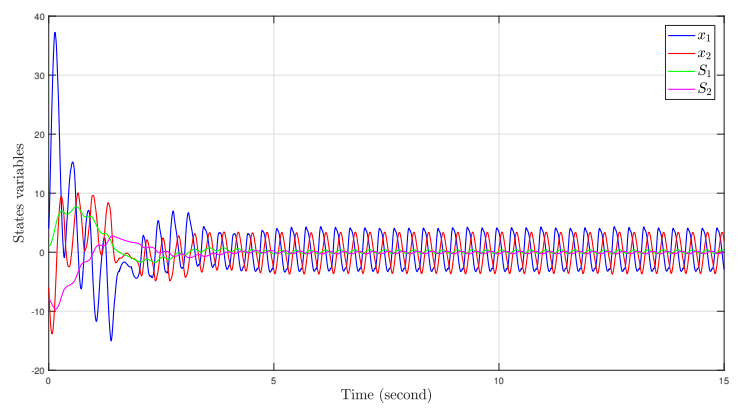

Fig. 1. Transient response of state variables for Example 1

Case i : $10^{2 i-1}\left[\begin{array}{llll}0.4 & -0.6 & 0.1 & -0.8\end{array}\right]$ for the master network, and $10^{2 i-1} \times\left[\begin{array}{llll}-0.6 & 0.4 & 1.1 & 0.2\end{array}\right]$ for the slave network, where $i=1,3,5$.

We fix the controller parameters as follows: $\rho_{1}=4, \rho_{2}=$ $8, \eta_{1}=0.8, \eta_{2}=1.2, \gamma_{1}=\gamma_{2}=0.1, p=0.5, q=1.5$. Then, from Theorem 1 and Theorem 2 we deduce that the networks in Example 1 are globally fixed-time synchronized, moreover, an upper-bound on the settling-time can be computed as $T_{1} \approx 51.07$ and $T_{2} \approx 64.92$. Fig. 2 depicts the time responses of the state variables as well as the synchronization error state variable. It confirms that the proposed method leads to the global fixed-time synchronization independently on the choice of the initial states. Moreover, one can see that the synchronization is achieved before $T_{1} \approx 51.07$.

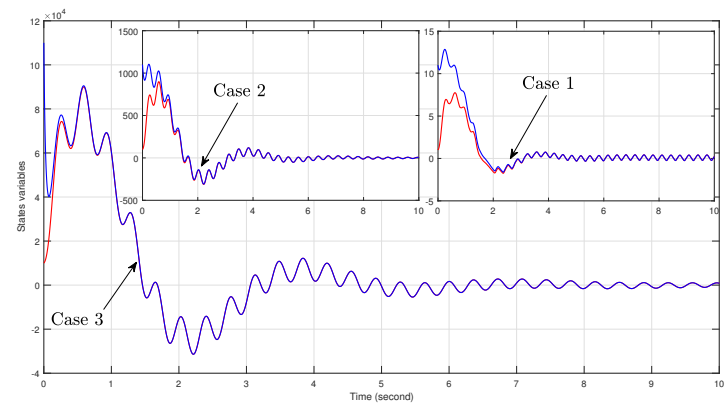

(a) Transient response of parts of state variables

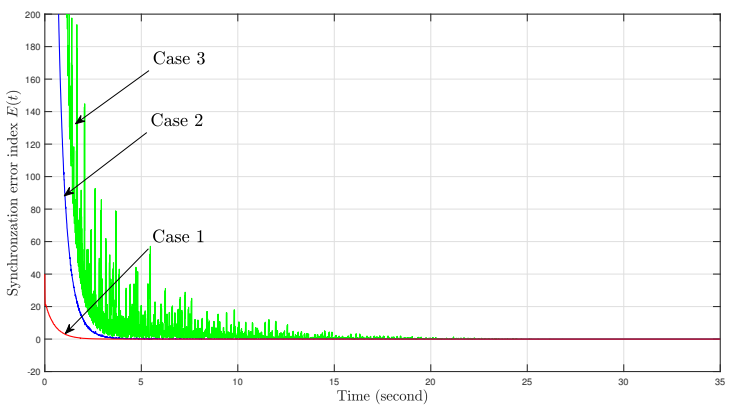

(b) Transient response of synchronization error state variable

Fig. 2. Simulation results of Example 1 with the proposed synchronization method

To better justify the interest of the fixed-time synchronization, we numerically compare our methodology with respect 
to the ones in [8], [15]. Table I summarizes the comparison w.r.t. the adaptive control method used in [8] which achieves the asymptotic synchronization of MTSCNNs and w.r.t. the synchronization method employed in [15] that ensures the finite time synchronization. As mentioned along the manuscript the settling-time for finite-time synchronization depends on the initial states and grows unbounded when the initial conditions go further away from the synchronization manifold. Besides, we point out that the results in [8], [15] require the perfect knowledge of the parameter $\varepsilon$ while our methodology needs only an upper-bound on it.

TABLE I

COMPARISONS OF THE SETTLING TIME

\begin{tabular}{c|c|c|c|c}
\hline \hline & {$[8]$} & {$[15]$} & Theorem 1 & Theorem 2 \\
\hline Case 1 & $+\infty$ & $40 \mathrm{sec}$ & $51.07 \mathrm{sec}$ & $64.92 \mathrm{sec}$ \\
\hline Case 2 & $+\infty$ & $4000 \mathrm{sec}$ & $51.07 \mathrm{sec}$ & $64.92 \mathrm{sec}$ \\
\hline Case 3 & $+\infty$ & $400000 \mathrm{sec}$ & $51.07 \mathrm{sec}$ & $64.92 \mathrm{sec}$ \\
\hline \hline
\end{tabular}

\section{CONCLUSiON}

In this paper, the fixed-time synchronization problem of MTSCNNs has been studied, and the obtained results show how the singular perturbation parameter affects the settlingtime. The validity of the obtained results has also been numerically tested by simulations. The future works will focus on the design of an event-triggered control method [23]-[25] or reinforcement learning method [26] to achieve the fixed-time synchronization and investigate the fixed-time synchronization of MTSCNNs with time-delay.

\section{REFERENCES}

[1] A. Meyer-Bäse, F. Ohl, and H. Scheich, "Singular perturbation analysis of competitive neural networks with different time scales," Neural Computation, vol. 8, no. 8, pp. 1731-1742, 1996.

[2] P. Kokotovic, H. K. Khalil, and J. O'reilly, Singular Perturbation Methods in Control: Analysis and Design. Philadelphia, PA: SIAM, 1999.

[3] X. Lou and B. Cui, "Synchronization of competitive neural networks with different time scales," Physica A: Statistical Mechanics and its Applications, vol. 380, pp. 563-576, 2007.

[4] X. Yang, C. Huang, and J. Cao, "An LMI approach for exponential synchronization of switched stochastic competitive neural networks with mixed delays," Neural Computing and Applications, vol. 21, no. 8, pp. 2033-2047, 2012

[5] Q. Gan, R. Hu, and Y. Liang, "Adaptive synchronization for stochastic competitive neural networks with mixed time-varying delays," Соттиnications in Nonlinear Science and Numerical Simulation, vol. 17, no. 9, pp. 3708-3718, 2012.

[6] A. Arbi, J. Cao, and A. Alsaedi, "Improved synchronization analysis of competitive neural networks with time-varying delays," Nonlinear Anal., Model. Control, vol. 23, no. 1, pp. 82-102, 2018.

[7] A. Pratap, R. Raja, J. Cao, G. Rajchakit, and H. M. Fardoun, "Stability and synchronization criteria for fractional order competitive neural networks with time delays: An asymptotic expansion of mittag leffler function," Journal of the Franklin Institute, vol. 356, no. 4, pp. 2212 2239, 2019.

[8] H. Gu, "Adaptive synchronization for competitive neural networks with different time scales and stochastic perturbation," Neurocomputing, vol. 73, no. 1-3, pp. 350-356, 2009.

[9] Q. Gan, "Synchronization of competitive neural networks with different time scales and time-varying delay based on delay partitioning approach," International Journal of Machine Learning and Cybernetics, vol. 4, no. 4, pp. 327-337, 2013
[10] Y. Shi and P. Zhu, "Synchronization of memristive competitive neural networks with different time scales," Neural Computing and Applications, vol. 25, no. 5, pp. 1163-1168, 2014.

[11] F. T. Duan and B. T. Cui, "Synchronization of memristor-based competitive neural networks with different time scales," in Applied Mechanics and Materials, vol. 740, pp. 238-242, Trans Tech Publ, 2015.

[12] S. Gong, S. Yang, Z. Guo, and T. Huang, "Global exponential synchronization of memristive competitive neural networks with time-varying delay via nonlinear control," Neural Processing Letters, vol. 49, no. 1, pp. 103-119, 2019.

[13] Y. Shi and P. Zhu, "Synchronization of stochastic competitive neural networks with different timescales and reaction-diffusion terms," Neural Computation, vol. 26, no. 9, pp. 2005-2024, 2014.

[14] X. Yang, J. Cao, Y. Long, and W. Rui, "Adaptive lag synchronization for competitive neural networks with mixed delays and uncertain hybrid perturbations," IEEE Transactions on Neural Networks, vol. 21, no. 10, pp. 1656-1667, 2010.

[15] Y. Li, X. Yang, and L. Shi, "Finite-time synchronization for competitive neural networks with mixed delays and non-identical perturbations," Neurocomputing, vol. 185, pp. 242-253, 2016

[16] L. Duan, X. Fang, X. Yi, and Y. Fu, "Finite-time synchronization of delayed competitive neural networks with discontinuous neuron activations," International Journal of Machine Learning and Cybernetics, vol. 9, no. 10, pp. 1649-1661, 2018.

[17] Y. Zou, X. Yang, R. Tang, and Z. Cheng, "Finite-time quantized synchronization of coupled discontinuous competitive neural networks with proportional delay and impulsive effects," Journal of the Franklin Institute, vol. 357, no. 16, pp. 11136-11152, 2020.

[18] A. Polyakov, "Nonlinear feedback design for fixed-time stabilization of linear control systems," IEEE Transactions on Automatic Control, vol. 57, no. 8, pp. 2106-2110, 2011.

[19] Y. Pan, P. Du, H. Xue, and H.-K. Lam, "Singularity-free fixed-time fuzzy control for robotic systems with user-defined performance," IEEE Transactions on Fuzzy Systems. DOI: 10.1109/TFUZZ.2020.2999746, 2020.

[20] L. Wang, Z. Zeng, J. Hu, and X. Wang, "Controller design for global fixed-time synchronization of delayed neural networks with discontinuous activations," Neural Networks, vol. 87, pp. 122-131, 2017.

[21] X. Liu and T. Chen, "Finite-time and fixed-time cluster synchronization with or without pinning control," IEEE Transactions on Cybernetics, vol. 48, no. 1, pp. 240-252, 2016.

[22] L. M. Pecora and T. L. Carroll, "Synchronization in chaotic systems," Physical Review Letters, vol. 64, no. 8, p. 821, 1990.

[23] H. Liang, X. Guo, Y. Pan, and T. Huang, "Event-triggered fuzzy bipartite tracking control for network systems based on distributed reduced-order observers," IEEE Transactions on Fuzzy Systems. DOI: 10.1109/TFUZZ.2020.2982618, 2020.

[24] Z. Zhu, Y. Pan, Q. Zhou, and C. Lu, "Event-triggered adaptive fuzzy control for stochastic nonlinear systems with unmeasured states and unknown backlash-like hysteresis," IEEE Transactions on Fuzzy Systems. DOI: 10.1109/TFUZZ.2020.2973950, 2020.

[25] H. Ma, H. Li, R. Lu, and T. Huang, "Adaptive event-triggered control for a class of nonlinear systems with periodic disturbances," SCIENCE CHINA Information Sciences, vol. 63, no. 5, p. 150212, 2020.

[26] H. Li, Y. Wu, and M. Chen, "Adaptive fault-tolerant tracking control for discrete-time multi-agent systems via reinforcement learning algorithm,' IEEE Transactions on Cybernetics. DOI: 10.1109/TCYB.2020.2982168, 2020. 